\title{
APPROXIMATE CONTROLLABILITY FOR A LINEAR MODEL OF FLUID STRUCTURE INTERACTION*
}

\author{
Axel Osses ${ }^{1}$ And Jean-Pierre Puel ${ }^{2}$
}

\begin{abstract}
We consider a linear model of interaction between a viscous incompressible fluid and a thin elastic structure located on a part of the fluid domain boundary, the other part being rigid. After having given an existence and uniqueness result for the direct problem, we study the question of approximate controllability for this system when the control acts as a normal force applied to the structure. The case of an analytic boundary has been studied by Lions and Zuazua in [9] where, in particular, a counterexample is given when the fluid domain is a ball. We prove a result of approximate controllability in the $2 d$-case when the rigid and the elastic parts of the boundary make a rectangular corner and if the control acts on the whole elastic structure.
\end{abstract}

Résumé. Nous considérons un modèle linéaire d'interaction entre un fluide visqueux incompressible et une structure élastique mince située sur une partie de la frontière du domaine fluide, l'autre partie de la frontière étant rigide. Après avoir donné un résultat d'existence et d'unicité pour le problème direct, nous étudions la question de la contrôlabilité approchée pour ce système lorsque le contrôle agit comme une force normale appliquée à la structure. Le cas d'une frontière analytique a été étudié par Lions et Zuazua dans [9] où, en particulier, un contre exemple est donné lorsque le domaine fluide est une boule. Nous montrons un résultat de contrôlabilité approchée dans le cas $2 d$ quand les parties rigide et élastique de la frontière forment un angle droit et si le contrôle agit sur toute la structure élastique.

AMS Subject Classification. 35B37, 35B60, 76D07, 73K50.

Received December 12, 1998. Revised June 30, 1999.

\section{INTRODUCTION}

We consider a viscous incompressible fluid contained in a domain $\Omega$ of $\mathbb{R}^{N}$ ( $N=2$ or 3 ), the boundary of which, denoted by $\Gamma$, is made of two parts: a rigid part $\Gamma_{R}$ and a part $\Gamma_{E}$ which is a thin elastic structure (membrane or shell). We consider a possibility of action (or control) on this system via a force $h$ acting on a part $\gamma$ of $\Gamma_{E}$, normal to the boundary.

Keywords and phrases: Controllability, fluid-structure interaction, nonsmooth domains, unique continuation property.

* The research of the first author has been supported by Fondap, Programa Mecánica Matemática. He also acknowledges the support of the European Alfa Project number 5.0199.9 and the French Scientific Committee ECOS.

${ }^{1}$ Universidad de Chile, Departamento de Ingeniería Matemática, Casilla 170/3, Correo 3, Santiago, Chile;

e-mail: axosses@dim.uchile.cl

${ }^{2}$ Université de Versailles Saint-Quentin and Centre de Mathématiques Appliquées, École Polytechnique, 91128 Palaiseau Cedex,

France; e-mail: jppuel@cmapx.polytechnique.fr

(c) EDP Sciences, SMAI 1999 
The movements of the fluid and of the elastic part of the boundary are coupled and therefore, the force $h$ has an effect on the fluid motion. We are interested here in studying this effect for some simplified models. More specifically, we study the question of approximate controllability for the fluid velocity (and for the deformation of the elastic structure) under the action of control $h$, in the case where the fluid motion is described by the Stokes system coupled with a linear elastodynamic model for the elastic boundary $\Gamma_{E}$. The question of approximate controllability means the following: given any neighborhood of any triple $\left(\mathbf{z}_{d}, \varphi_{d}, \psi_{d}\right)$ representing the desired fluid velocity, structure displacement and structure velocity in a convenient space (which will be made precise later on), can we choose $h$ in a suitable space such that, at final time $T$, the triple $\left(\mathbf{y}(T), w(T), \frac{\partial w(T)}{\partial t}\right)$ of the fluid velocity, structure displacement and structure velocity, lies in the given neighborhood.

Very few results are known on these questions, even for simple models. The answers seem to depend strongly on the geometry or regularity of the domain. In [9], Lions and Zuazua give a counterexample to approximate controllability when $\Omega$ is a ball. In a previous work [11], we proved that when $\Omega$ is a $2 d$-rectangle and $\gamma=\Gamma_{E}$ is the union of two opposite sides, then we have approximate controllability. Here, we show that in the $2 d$-case, when $\gamma=\Gamma_{E}$ and the joint between $\Gamma_{E}$ and $\Gamma_{R}$ contains an exact rectangular corner (which includes the previous case) then again we have approximate controllability.

Of course these results are very specific and partial for a problem which has a lot of practical interest in its general setting. When the fluid motion is governed by Navier-Stokes equations and the elastic structure displacement by nonlinear elastodynamic equations, we have, at the moment, no result at all.

An interesting question is also to consider our linear system in the case where the control is performed via the action of piezoelectric patches on the elastic part of the boundary, and to study what part of the fluid velocity one can control by this mean. Again, this problem is completely open at the moment.

Our article is organized as follows:

in Section 2 we give the precise model that we consider (in a general geometry) with a correct mathematical setting and we give the standard preliminary results;

in Section 3 we give our precise result and we give a first step of the proof following [7] and pointing out the main difficulties and open questions.

in Section 4 we prove an auxiliary result on unique continuation for an eigenvalue problem and this will complete the proof of our approximate controllability result.

\section{MATHEMATICAL MODEL AND PRELIMINARY RESUlts}

We consider a bounded connected open set $\Omega$ of $\mathbb{R}^{N}(N=2$ or 3$)$ with boundary $\Gamma=\bar{\Gamma}_{R} \cup \bar{\Gamma}_{E}$ where $\Gamma_{R}$ and $\Gamma_{E}$ are two non-empty relatively open subsets of $\Gamma$. For simplicity, we assume that each of $\Gamma_{R}$ and $\Gamma_{E}$ is regular, the joint between them being either smooth or convex corners. We denote by $\mathbf{n}$ the outward unit normal vector at a point of $\Gamma$ and by $\tau$ (if $N=2$ ) or $\left(\tau^{1}, \tau^{2}\right)$ (if $N=3$ ) the unit tangent vector(s) (orthogonal if $N=3$ ) which generate the tangent (plane) to $\Gamma$.

In $\Omega$ we have an incompressible viscous fluid with viscosity $\nu>0$. Velocity $\mathbf{y}$ and pressure $p$ of the fluid satisfy the Stokes system. On the rigid part of the boundary denoted by $\Gamma_{R}$, the fluid satisfies the no-slip boundary conditions.

We denote by $\sigma(\mathbf{y}, p)$ the stress tensor

$$
\sigma_{i j}(\mathbf{y}, p)=-p \delta_{i j}+2 \nu e_{i j}(\mathbf{y})
$$

where

$$
e_{i j}=\frac{1}{2}\left(\frac{\partial y_{i}}{\partial x_{j}}+\frac{\partial y_{j}}{\partial x_{i}}\right)
$$

The elastic part of the boundary, denoted by $\Gamma_{E}$, can be an elastic membrane or shell (arch). In order to simplify the presentation, we take here the case of a shell (arch) that we suppose clamped on its edges. We 
only consider the deflection $w$ of the structure (displacement normal to $\Gamma_{E}$ ) which is then a function of time $t$ with values in $H_{0}^{2}\left(\Gamma_{E}\right)$ The stored elastic energy is represented by a quadratic form $b(w, w)$ associated with a symmetric bilinear form $b(w, \widetilde{w})$ and a fourth order differential operator $\mathbf{B} w$ such that

$$
\forall w, \widetilde{w} \in H_{0}^{2}\left(\Gamma_{E}\right), \quad b(w, \widetilde{w})=\int_{\Gamma_{E}} \mathbf{B} w \cdot \widetilde{w} d \Gamma
$$

We assume a coercivity hypothesis on $b$, i.e.

$$
\exists \alpha>0, \forall w \in H_{0}^{2}\left(\Gamma_{E}\right), \quad b(w, w) \geq \alpha\|w\|_{H_{0}^{2}\left(\Gamma_{E}\right)}^{2} .
$$

The control variable $h$ is a density of force, normal to $\Gamma_{E}$, acting on a non-empty open part $\gamma$ of $\Gamma_{E}$ and we write $\chi_{\gamma}$ for the characteristic function of $\gamma$.

Coupling between the fluid motion and the elastic structure displacement is performed via continuity of (normal) velocities and the normal force $\sigma(\mathbf{y}, p) \mathbf{n} \cdot \mathbf{n}$ induced by the fluid on $\Gamma_{E}$. Let us notice that $\sigma(\mathbf{y}, p) \mathbf{n} \cdot \mathbf{n}$ is defined, as the pressure, up to the addition of a constant in the space variable (which may depend on time).

We then obtain a full set of equations describing the motion of our system, complemented by initial condition.

$$
\begin{aligned}
& \frac{\partial \mathbf{y}}{\partial t}-\nu \Delta \mathbf{y}+\nabla p=0 \quad \text { in } \quad \Omega \times(0, T) \\
& \operatorname{div} \mathbf{y}=0 \quad \text { in } \Omega \times(0, T) \\
& \mathbf{y}=0 \quad \text { on } \quad \Gamma_{R} \times(0, T) \\
& \mathbf{y} \cdot \tau=0 \quad\left(\text { or } \mathbf{y} \cdot \tau^{i}=0, i=1,2\right) \text { on } \Gamma_{E} \times(0, T) \\
& \mathbf{y} \cdot \mathbf{n}=\frac{\partial w}{\partial t} \quad \text { on } \quad \Gamma_{E} \times(0, T) \\
& \mathbf{y}(0)=\mathbf{y}_{0} \quad \text { in } \Omega \\
& \frac{\partial^{2} w}{\partial t^{2}}+\mathbf{B} w=-\sigma(\mathbf{y}, p) \mathbf{n} \cdot \mathbf{n}+h \cdot \chi_{\gamma} \quad \text { on } \quad \Gamma_{E} \times(0, T) \\
& w(t) \in H_{0}^{2}\left(\Gamma_{E}\right) \quad \text { a.e. in } \quad(0, T) \\
& w(0)=w_{0}, \frac{\partial w}{\partial t}(0)=w_{1} \quad \text { in } \quad \Gamma_{E} .
\end{aligned}
$$

We now have to give a precise mathematical formulation of problem (2.5-2.6) and an existence result for the direct problem.

We define the spaces

$$
\begin{aligned}
& V=\left\{\mathbf{z} \in H^{1}(\Omega)^{N}, \operatorname{div} \mathbf{z}=0, \mathbf{z}=\mathbf{0} \text { on } \Gamma_{R},\right. \\
& \left.\mathbf{z} \cdot \tau=0 \text { on } \Gamma_{E} \quad\left(\text { or } \mathbf{z} \cdot \tau^{i}=0, i=1,2 \text { on } \Gamma_{E}\right)\right\} \\
& H=\left\{\mathbf{z} \in L^{2}(\Omega)^{N}, \operatorname{div} \mathbf{z}=0, \mathbf{z} \cdot \mathbf{n}=0 \text { on } \Gamma_{R}\right\} \\
& X=\left\{(\mathbf{z}, \varphi, \psi) \in V \times H_{0}^{2}\left(\Gamma_{E}\right) \times L^{2}\left(\Gamma_{E}\right), \mathbf{z} \cdot n=\psi \text { on } \Gamma_{E}\right\} \text {. }
\end{aligned}
$$

Notice that when $(\mathbf{z}, \varphi, \psi) \in X$, as a consequence of the fact that $\operatorname{div} \mathbf{z}=0$ in $\Omega$ we have

$$
\int_{\Gamma_{E}} \psi d \Gamma=0
$$


Therefore $X$ is a subset of $V \times H_{0}^{2}\left(\Gamma_{E}\right) \times L_{0}^{2}\left(\Gamma_{E}\right)$, where

$$
L_{0}^{2}\left(\Gamma_{E}\right)=\left\{\psi \in L^{2}\left(\Gamma_{E}\right), \int_{\Gamma_{E}} \psi d \Gamma=0\right\}
$$

Because of (2.4), we can suppose that $H_{0}^{2}\left(\Gamma_{E}\right)$ is equipped with the scalar product $b(w, \widetilde{w})$.

Let $\mathbf{A}$ be the unbounded operator in $H \times H_{0}^{2}\left(\Gamma_{E}\right) \times L_{0}^{2}\left(\Gamma_{E}\right)$ defined as follows:

$$
\begin{array}{r}
\mathbf{D}(\mathbf{A})=\left\{(\mathbf{z}, \varphi, \psi) \in X, \exists q \in L^{2}(\Omega),-\nu \Delta \mathbf{z}+\nabla q \in L^{2}(\Omega)^{N},\right. \\
\left.\sigma(\mathbf{z}, q) \mathbf{n} \cdot \mathbf{n} \in L^{2}\left(\Gamma_{E}\right), \mathbf{B} \varphi \in L^{2}\left(\Gamma_{E}\right), \psi \in H_{0}^{2}\left(\Gamma_{E}\right)\right\}, \\
\forall(\mathbf{z}, \varphi, \psi) \in \mathbf{D}(\mathbf{A}), \mathbf{A}\left(\begin{array}{c}
\mathbf{z} \\
\varphi \\
\psi
\end{array}\right)=\left(\begin{array}{c}
-\nu \Delta \mathbf{z}+\nabla q \\
-\psi \\
\sigma(\mathbf{z}, q) \mathbf{n} \cdot \mathbf{n}+\mathbf{B} \varphi
\end{array}\right) .
\end{array}
$$

Notice that when $\mathbf{z} \in V$ and $-\nu \Delta z+\nabla q \in L^{2}(\Omega)^{N}$, then as in [8] one can define $\sigma(\mathbf{z}, q) \mathbf{n} \cdot \mathbf{n}$, up to a constant, in $H^{-\frac{1}{2}}(\Gamma)$.

Lemma 2.1. The operator $\mathbf{A}$ is maximal monotone.

Proof. For $\lambda>0$ and $\varepsilon>0$, we consider the bilinear form

$$
a_{\lambda, \varepsilon}[(\mathbf{z}, \varphi, \psi),(\widetilde{\mathbf{z}}, \widetilde{\varphi}, \widetilde{\psi})]=\nu \int_{\Omega} \nabla \mathbf{z} \cdot \nabla \widetilde{\mathbf{z}} d x+\lambda \int_{\Omega} \mathbf{z} \cdot \widetilde{\mathbf{z}} d x+\lambda b(\varphi, \widetilde{\varphi})+\varepsilon b(\psi, \widetilde{\psi})+\lambda \int_{\Gamma_{E}} \psi \cdot \widetilde{\psi} d \Gamma
$$

on the space

$$
Y=\left\{(\mathbf{z}, \varphi, \psi) \in X, \psi \in H_{0}^{2}\left(\Gamma_{E}\right)\right\}
$$

For every $(\mathbf{f}, g, k) \in H \times H_{0}^{2}\left(\Gamma_{E}\right) \times L_{0}^{2}\left(\Gamma_{E}\right)$, there exists a unique $\left(\mathbf{z}^{\varepsilon}, \varphi^{\varepsilon}, \psi^{\varepsilon}\right) \in Y$ such that

$$
a_{\lambda, \varepsilon}\left[\left(\mathbf{z}^{\varepsilon}, \varphi^{\varepsilon}, \psi^{\varepsilon}\right),(\widetilde{\mathbf{z}}, \widetilde{\varphi}, \widetilde{\psi})\right]=(\mathbf{f}, \widetilde{\mathbf{z}})_{H}+b(g, \widetilde{\varphi})+\int_{\Gamma_{E}} k \widetilde{\psi} d \Gamma, \quad \forall(\widetilde{\mathbf{z}}, \widetilde{\varphi}, \widetilde{\psi}) \in Y .
$$

Moreover, $\left(\mathbf{z}^{\varepsilon}, \varphi^{\varepsilon}, \psi^{\varepsilon}\right) \in \mathbf{D}(\mathbf{A})$.

In fact, this corresponds to solving the regularized problem

$$
\mathbf{A}_{\varepsilon}\left(\begin{array}{c}
\mathbf{z}^{\varepsilon} \\
\varphi^{\varepsilon} \\
\psi^{\varepsilon}
\end{array}\right)+\lambda\left(\begin{array}{c}
\mathbf{z}^{\varepsilon} \\
\varphi^{\varepsilon} \\
\psi^{\varepsilon}
\end{array}\right)=\left(\begin{array}{c}
\mathbf{f} \\
g \\
k
\end{array}\right)
$$

where

$$
\mathbf{A}_{\varepsilon}=\mathbf{A}+\left(\begin{array}{ccc}
0 & 0 & 0 \\
0 & 0 & 0 \\
0 & 0 & \varepsilon \mathbf{B}
\end{array}\right)
$$

Now letting $\varepsilon$ tend to 0 it is easy to see that

$$
\left(\mathbf{z}^{\varepsilon}, \varphi^{\varepsilon}, \psi^{\varepsilon}\right) \rightarrow(\mathbf{z}, \varphi, \psi) \quad \text { in } \quad X \text {-weakly, }
$$

with $\psi^{\varepsilon} \rightarrow \psi$ in $H_{0}^{2}\left(\Gamma_{E}\right)$-weakly, $(\mathbf{z}, \varphi, \psi) \in \mathbf{D}(\mathbf{A})$, and $(\mathbf{z}, \varphi, \psi)$ satisfies

$$
\mathbf{A}\left(\begin{array}{l}
\mathbf{z} \\
\varphi \\
\psi
\end{array}\right)+\lambda\left(\begin{array}{l}
\mathbf{z} \\
\varphi \\
\psi
\end{array}\right)=\left(\begin{array}{l}
\mathbf{f} \\
g \\
k
\end{array}\right)
$$


This, together with the fact that $\mathbf{A}$ is positive, completes the proof of Lemma 2.1.

We now consider the following problem

$$
\begin{gathered}
\frac{d}{d t}\left(\begin{array}{c}
\mathbf{y} \\
w \\
\eta
\end{array}\right)+\mathbf{A}\left(\begin{array}{c}
\mathbf{y} \\
w \\
\eta
\end{array}\right)=\left(\begin{array}{c}
0 \\
0 \\
h \chi_{\gamma}
\end{array}\right) \\
\mathbf{y}(0)=\mathbf{y}_{0}, w(0)=w_{0}, \eta(0)=w_{1} .
\end{gathered}
$$

Theorem 2.2. If $\mathbf{y}_{0} \in H, w_{0} \in H_{0}^{2}\left(\Gamma_{E}\right), w_{1} \in L_{0}^{2}\left(\Gamma_{E}\right)$ and if $h$ belongs to $L^{1}\left(0, T ; L^{2}(\gamma)\right)$, there exists a unique solution $(\mathbf{y}, w, \eta)$ of $(2.11)$ with

$$
\begin{gathered}
(\mathbf{y}, w, \eta) \in C\left([0, T] ; H \times H_{0}^{2}\left(\Gamma_{E}\right) \times L_{0}^{2}\left(\Gamma_{E}\right)\right), \\
\mathbf{y} \in L^{2}(0, T ; V), \\
\mathbf{y} \cdot \mathbf{n}=\eta \quad \text { on } \quad \Gamma_{E} \times(0, T) .
\end{gathered}
$$

Moreover $\eta=\frac{\partial w}{\partial t}$ and $(\mathbf{y}, w)$ is solution (in a weak sense) of (2.5-2.6).

Proof. From Lemma 2.1 we see (cf. [4] or [12]) that $\mathbf{D}(\mathbf{A})$ is dense in $H \times H_{0}^{2}\left(\Gamma_{E}\right) \times L_{0}^{2}\left(\Gamma_{E}\right)^{N}$, that $\mathbf{A}$ is generator of a continuous semigroup of contractions and therefore, if $\left(\mathbf{y}_{0}, w_{0}, w_{1}\right) \in \mathbf{D}(\mathbf{A})$ and $h \in \mathbf{D}(] 0, T\left[; L^{2}(\gamma)\right)$, there exists a unique solution $(\mathbf{y}, w, \eta)$ of $(2.11)$ with $(\mathbf{y}, w, \eta) \in C([0, T] ; \mathbf{D}(\mathbf{A}))$ and $\eta=\frac{\partial w}{\partial t}$.

Moreover, multiplying (2.11) by $(\mathbf{y}, w, \eta)$ in $H \times H_{0}^{1}\left(\Gamma_{E}\right) \times L_{0}^{2}\left(\Gamma_{E}\right)$, using (2.7-2.8), we obtain (with the usual convention of summation for repeated indices)

$$
\begin{aligned}
\frac{1}{2} \frac{d}{d t}|\mathbf{y}|_{H}^{2}+\frac{1}{2} \frac{d}{d t}|\eta|_{L^{2}\left(\Gamma_{E}\right)}^{2} & +\frac{1}{2} \frac{d}{d t} b(w, w)+2 \nu \int_{\Omega} e_{i j}(\mathbf{y}) e_{i j}(\mathbf{y}) d x \\
& -\int_{\Gamma} \sigma(\mathbf{y}, p) \mathbf{n} \cdot \mathbf{y} d \Gamma+\int_{\Gamma_{E}} \sigma(\mathbf{y}, p) \mathbf{n} \cdot \mathbf{n} \eta d \Gamma=\int_{\gamma} h \eta d \Gamma .
\end{aligned}
$$

Using the definition of the space $X$ and the second equation in (2.11a) we have $\mathbf{y}=\mathbf{0}$ on $\Gamma_{R} \times(0, T), \mathbf{y} \cdot \tau=0$ (or $\left.\mathbf{y} \cdot \tau_{i}=0, i=1,2\right)$ on $\Gamma_{E} \times(0, T)$ and $\mathbf{y} \cdot \mathbf{n}=\eta=\frac{\partial w}{\partial t}$ on $\Gamma_{E} \times(0, T)$. From equality (2.12) we then obtain the following energy estimate:

$$
\frac{1}{2} \frac{d}{d t}|\mathbf{y}|_{H}^{2}+\frac{1}{2} \frac{d}{d t}\left|\frac{\partial w}{\partial t}\right|_{L^{2}\left(\Gamma_{E}\right)}^{2}+\frac{1}{2} \frac{d}{d t} b(w, w)+2 \nu \int_{\Omega}|e(\mathbf{y})|^{2} d x=\int_{\gamma} h \frac{\partial w}{\partial t} d \Gamma .
$$

From the semigroup properties, or directly from the energy estimate above, we see that there exists $C>0$ such that

$$
\begin{aligned}
\|\mathbf{y}\|_{C([0, T] ; H)}^{2}+ & \left\|\frac{\partial w}{\partial t}\right\|_{C\left([0, T] ; L^{2}\left(\Gamma_{E}\right)\right)}^{2}+\|w\|_{C\left([0, T] ; H_{0}^{2}\left(\Gamma_{E}\right)\right)}^{2}+\|\mathbf{y}\|_{L^{2}(0, T ; V)}^{2} \\
& \leq C\left(\left|\mathbf{y}_{0}\right|_{H}^{2}+\left\|w_{0}\right\|_{H_{0}^{2}\left(\Gamma_{E}\right)}^{2}+\left|w_{1}\right|_{L_{0}^{2}\left(\Gamma_{E}\right)}^{2}+\|h\|_{L^{1}\left(0, T ; L^{2}(\gamma)\right)}^{2}\right) .
\end{aligned}
$$

Now if $\mathbf{y}_{0} \in H, w_{0} \in H_{0}^{2}\left(\Gamma_{E}\right), w_{1} \in L_{0}^{2}\left(\Gamma_{E}\right)$ and $h \in L^{1}\left(0, T ; L^{2}(\gamma)\right)$ we take approximation sequences $\left(\mathbf{y}_{0}^{k}, w_{0}^{k}, w_{1}^{k}\right) \in \mathbf{D}(\mathbf{A}), h^{k} \in \mathbf{D}(] 0, T\left[; L^{2}(\gamma)\right)$ such that

$$
\begin{aligned}
\left(\mathbf{y}_{0}^{k}, w_{0}^{k}, w_{1}^{k}\right) & \rightarrow\left(\mathbf{y}_{0}, w_{0}, w_{1}\right) \quad \text { in } H \times H_{0}^{2}\left(\Gamma_{E}\right) \times L_{0}^{2}\left(\Gamma_{E}\right) \\
h^{k} & \rightarrow h \quad \text { in } L^{1}\left(0, T ; L^{2}(\gamma)\right) .
\end{aligned}
$$

For fixed $k$, there exists a unique solution $\left(\mathbf{y}^{k}, w^{k}, \eta^{k}\right)$ of $(2.11)$. 
Using linearity of (2.11) and (2.14), it is easy to show that $\left(\mathbf{y}^{k}, w^{k}, \eta^{k}\right)$ is a Cauchy sequence in $C([0, T] ; H \times$ $\left.H_{0}^{2}\left(\Gamma_{E}\right) \times L_{0}^{2}\left(\Gamma_{E}\right)\right)$ and that $\mathbf{y}^{k}$ is a Cauchy sequence in $L^{2}(0, T ; V)$. Therefore $\left(\mathbf{y}^{k}, w^{k}, \eta^{k}\right)$ converges to $(\mathbf{y}, w, \eta)$ in $C\left([0, T] ; H \times H_{0}^{2}\left(\Gamma_{E}\right) \times L_{0}^{2}\left(\Gamma_{E}\right)\right)$ and $\mathbf{y}^{k}$ converges to $\mathbf{y}$ in $L^{2}(0, T ; V)$.

It is easy to see that $\eta=\frac{\partial w}{\partial t}$ and $\mathbf{y} \cdot \mathbf{n}=\eta$ on $\Gamma_{E} \times(0, T)$.

In order to pass to the limit in the equation $(2.11)$, the only difficulty is to pass to the limit in $\sigma\left(\mathbf{y}^{k}, p^{k}\right) \mathbf{n} \cdot \mathbf{n}$. We have:

$$
\mathbf{y}^{k} \rightarrow \mathbf{y} \quad \text { in } \quad L^{2}(0, T ; V)-\nu \Delta \mathbf{y}^{k}+\nabla p^{k} \rightarrow-\nu \Delta \mathbf{y}+\nabla p \quad \text { in } \quad H^{-1}(0, T ; V)
$$

Therefore, at least,

$$
\sigma\left(\mathbf{y}^{k}, p^{k}\right) \mathbf{n} \cdot \mathbf{n} \rightarrow \sigma(\mathbf{y}, p) \mathbf{n} \cdot \mathbf{n} \quad \text { in } \quad H^{-1}\left(0, T ; H^{-\frac{1}{2}}(\Gamma)\right)
$$

and this completes the proof of Theorem 2.2 .

Remark 2.3. Because $\operatorname{div} \mathbf{y}=0$ in $\Omega$, the solution $(\mathbf{y}, w, \eta)$ of $(2.11)$ satisfies

$$
\forall t \in[0, T], \quad \int_{\Gamma_{E}} \eta(t) d \Gamma=0
$$

As $\eta=\frac{\partial w}{\partial t}$ we see that

$$
\forall t \in[0, T], \quad \int_{\Gamma_{E}} w(t) d \Gamma=\int_{\Gamma_{E}} w_{0} d \Gamma .
$$

\section{Approximate controllability Results}

In [9], Lions and Zuazua considered the previous problem in the case of a domain $\Omega$ with analytic boundary and $\gamma=\Gamma_{E}$. They showed that approximate controllability is valid when the eigenvalues of the Dirichlet problem in $\Omega$ are all simple and they gave a counterexample to approximate controllability when $\Omega$ is a ball.

Here, we want to study the case where $\Gamma$ is not analytic (in particular when it contains a flat part) and the situation is quite different. We give a positive result of approximate controllability when $\Omega$ has a special geometry in dimension 2 and we point out the main difficulties in the general case.

Because of Remark 2.3, we introduce the following linear and affine subspaces:

$$
\begin{aligned}
& W_{0}=\left\{w \in H_{0}^{2}\left(\Gamma_{E}\right), \int_{\Gamma_{E}} w d \Gamma=0\right\} \\
& W=w_{0}+W_{0}
\end{aligned}
$$

Theorem 3.1. We suppose that $N=2$ and that $\gamma=\Gamma_{E}$ (the control acts on the whole elastic structure). Furthermore we assume that $\Gamma_{R}$ and $\Gamma_{E}$ are separately regular and that one junction point between $\Gamma_{E}$ and $\Gamma_{R}$, that we call $A$, is an exact rectangular corner, i.e. there exists a neighborhood $\mathbf{U}$ of $A$ such that $\left(\Gamma_{E} \cap \mathbf{U}\right)$ and $\left(\Gamma_{R} \cap \mathbf{U}\right)$ are two sides of a rectangular angle with vertex $A$.

Then problem (2.5-2.6) is approximately controllable in $H \times W \times L_{0}^{2}\left(\Gamma_{E}\right)$, i.e. for every $\left(\mathbf{y}_{0}, w_{0}, w_{1}\right) \in$ $H \times H_{0}^{2}\left(\Gamma_{E}\right) \times L_{0}^{2}\left(\Gamma_{E}\right)$, the set of reachable states

$$
R(T)=\left\{\left(\mathbf{y}(T), w(T), \frac{\partial w}{\partial t}(T)\right), h \in L^{1}\left(0, T ; L^{2}(\gamma)\right)\right\}
$$

is dense in $H \times W \times L_{0}^{2}\left(\Gamma_{E}\right)$. 


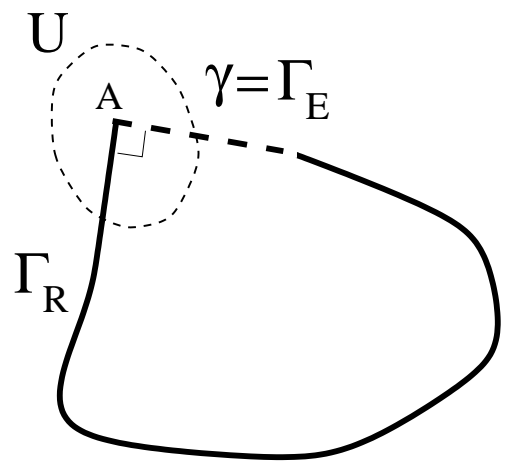

FigurE 1. Shape of a domain satisfying conditions of Theorem 3.1.

Proof. (First part. Reduction to a unique continuation problem). In this section we give the first part of the proof where we do not take into account the specific hypothesis of Theorem 3.1 in order to show the difficulties arising in the general problem. In Section 4 we present the second and final part of this proof.

As we deal with a linear problem, using a simple translation, we only have to consider the case

$$
\mathbf{y}_{0}=\mathbf{0} ; w_{0}=0 ; w_{1}=0
$$

and we then have to show that $R(T)$ is dense in $H \times W_{0} \times L_{0}^{2}\left(\Gamma_{E}\right)$. Because of Remark 2.3 we know that $R(T)$ is a linear subspace of $H \times W_{0} \times L_{0}^{2}\left(\Gamma_{E}\right)$. Therefore we have to prove that its orthogonal $R(T)^{\perp}$ in $H \times W_{0} \times L_{0}^{2}\left(\Gamma_{E}\right)$ is reduced to $\{\mathbf{0}, 0,0\}$.

Let $\left(\mathbf{z}_{T}, \varphi_{T}, \psi_{T}\right) \in R(T)^{\perp}$ and let us solve the backward adjoint problem

$$
\begin{gathered}
-\frac{d}{d t}\left(\begin{array}{c}
\mathbf{z} \\
\varphi \\
\psi
\end{array}\right)+\mathbf{A}^{*}\left(\begin{array}{c}
\mathbf{z} \\
\varphi \\
\psi
\end{array}\right)=0 \\
\mathbf{z}(T)=\mathbf{z}^{T}, \varphi(T)=\varphi_{T}, \psi(T)=\psi_{T} .
\end{gathered}
$$

As the operator $A$ is maximal monotone, so is $A^{*}[4]$ and therefore (3.4) has a unique solution

$$
(\mathbf{z}, \varphi, \psi) \in C\left([0, T] ; H \times W_{0} \times L_{0}^{2}\left(\Gamma_{E}\right)\right)
$$

with

$$
\mathbf{z} \in L^{2}(0, T ; V), \mathbf{z} \cdot \mathbf{n}=\psi \text { on } \Gamma_{E} \times(0, T)
$$

and

$$
\psi=\frac{\partial \varphi}{\partial t}
$$

After an easy computation of $\mathbf{A}^{*}$, (3.4) can be interpreted as the following system

$$
\begin{aligned}
-\frac{\partial \mathbf{z}}{\partial t}-\nu \Delta \mathbf{z}+\nabla q & =0 \quad \text { in } \Omega \times(0, T) \\
\operatorname{div} \mathbf{z} & =0 \quad \text { in } \Omega \times(0, T) \\
\mathbf{z} & =0 \quad \text { on } \Gamma_{R} \times(0, T) \\
\mathbf{z} \cdot \tau & =0 \quad\left(\text { or } \mathbf{z} \cdot \tau^{i}=0, i=1,2\right) \quad \text { on } \Gamma_{E} \times(0, T) \\
\mathbf{z} \cdot \mathbf{n} & =\psi=\frac{\partial \varphi}{\partial t} \quad \text { on } \quad \Gamma_{E} \times(0, T) \\
\mathbf{z}(T) & =\mathbf{z}_{T} \quad \text { in } \Omega,
\end{aligned}
$$


and

$$
\begin{aligned}
-\frac{\partial \varphi}{\partial t}+\psi & =0 \quad \text { on } \quad \Gamma_{E} \times(0, T) \\
-\frac{\partial \psi}{\partial t}-\mathbf{B} \varphi+\sigma(\mathbf{z}, q) \mathbf{n} \cdot \mathbf{n} & =C \quad \text { on } \quad \Gamma_{E} \times(0, T) \\
\varphi(t) & \in H_{0}^{2}\left(\Gamma_{E}\right) \quad \text { a.e. in } \quad(0, T) \\
\varphi(T) & =\varphi_{T}, \psi(T)=\psi_{T} \quad \text { on } \quad \Gamma_{E},
\end{aligned}
$$

where $C$ is a function from $(0, T)$ with values in $\mathbb{R}$, since the left hand side in $(3.6 \mathrm{~b})$ results orthogonal to $L_{0}^{2}\left(\Gamma_{E}\right)$. This implies also that for each $t, C(t)$ is the same constant on all the connected components of $\Gamma_{E}$. We can eliminate $\psi=\frac{\partial \varphi}{\partial t}$ in (3.6) to obtain

$$
\begin{aligned}
\frac{\partial^{2} \varphi}{\partial t^{2}}+\mathbf{B} \varphi-\sigma(\mathbf{z}, q) \mathbf{n} \cdot \mathbf{n} & =C \quad \text { on } \quad \Gamma_{E} \times(0, T) \\
\varphi(t) & \in H_{0}^{2}\left(\Gamma_{E}\right)^{N} \quad \text { a.e. in } \quad(0, T) \\
\varphi(T) & =\varphi_{T}, \frac{\partial \varphi}{\partial t}(T)=\psi_{T} \quad \text { on } \quad \Gamma_{E} .
\end{aligned}
$$

Moreover, for every solution of (3.4) and every solution of (2.11) we have

$$
\begin{aligned}
\forall h & \in L^{1}\left(0, T ; L^{2}(\gamma)\right), \\
\left(\mathbf{y}(T), \mathbf{z}_{T}\right)+b\left(w(T), \varphi_{T}\right) & +\left(\frac{\partial w}{\partial t}(T), \psi_{T}\right)_{L^{2}\left(\Gamma_{E}\right)}=\int_{0}^{T} \int_{\gamma} h \psi d \Gamma d t .
\end{aligned}
$$

From (3.8), we see that $\left(\mathbf{z}_{T}, \varphi_{T}, \psi_{T}\right) \in R(T)^{\perp}$ is equivalent to

$$
\int_{0}^{T} \int_{\gamma} h \psi d \Gamma d t=0, \quad \forall h \in L^{1}\left(0, T ; L^{2}(\gamma)\right)
$$

or to

$$
\frac{\partial \varphi}{\partial t}=\psi=0 \quad \text { on } \quad \gamma \times(0, T) .
$$

Proving approximate controllability for the original problem is now equivalent to a unique continuation property, namely to proving that $(3.5,3.7,3.10)$ imply

$$
\mathbf{z}_{T}=\mathbf{0}, \varphi_{T}=\mathbf{0}, \psi_{T}=0 .
$$

The counterexample given in [9] when $\Omega$ is a ball shows that it is hopeless to prove the unique continuation property by local methods in the neighborhood of a regular point of $\gamma$.

When $\gamma \neq \Gamma_{E},(3.5,3.7,3.10)$ cannot be decoupled and it is a system of mixed type Stokes-Petrovskii. At the moment, we do not know any method to try to prove the unique continuation property in this case and the question is completely open.

When $\gamma=\Gamma_{E}$, system $(3.5,3.7,3.10)$ decouples. Because of $(3.10)$ we have

$$
\frac{\partial \varphi}{\partial t}=0 \quad \text { on } \quad \Gamma_{E} \times(0, T)
$$

and therefore

$$
\mathbf{z}=0 \quad \text { on } \quad \Gamma \times(0, T)
$$


From (3.5) $\mathbf{z}$ is then a solution of a Stokes system with homogeneous Dirichlet condition

$$
\begin{array}{rlrl}
-\frac{\partial \mathbf{z}}{\partial t}-\nu \Delta \mathbf{z}+\nabla q & =0 & \text { in } \quad & \Omega \times(0, T) \\
\operatorname{div} \mathbf{z} & =0 & \text { in } \quad & \Omega \times(0, T) \\
\mathbf{z} & =0 & \text { on } \quad \Gamma \times(0, T) \\
\mathbf{z}(T) & =\mathbf{z}_{T} & \text { in } \quad & \Omega .
\end{array}
$$

Let us remark that as $\mathbf{z} \in C([0, T] ; H)$ then we have $\mathbf{z}_{T} \cdot \mathbf{n}=0$ on $\Gamma$.

Since the Stokes operator generates an analytic semigroup [12], $\mathbf{z}$ and $\nabla q$ are real analytic functions of $t$ for $t<T$ with values in $H^{2}(\Omega)^{N} \cap H_{0}^{1}(\Omega)^{N} \times H^{1}(\Omega)$ under our regularity hypothesis on the domain.

Moreover, we can choose $q$ such that

$$
\int_{\Omega} q(t, x) d x=0, \quad t<T .
$$

This choice makes the function $q$ itself a real analytic function of $t$ for $t<T$ (this can be verified using the power expansion of $\nabla q$ and the Poincaré-Wirtinger inequality). Therefore, $\sigma(\mathbf{z}, q) \mathbf{n} \cdot \mathbf{n}$ is also a real analytic function of time $t$ for $t<T$ with values in $H^{\frac{1}{2}}(\Gamma)$ for example. This fact, together with condition (3.11) implies that the function $C$ in $(3.7 \mathrm{a})$ is a real analytic function from $(0, T)$ with values in $\mathbb{R}$. Then we can take the time derivative in (3.7a). Using the extra condition (3.11) we obtain

$$
\frac{\partial}{\partial t} \sigma(\mathbf{z}, q) \mathbf{n} \cdot \mathbf{n}=C_{0}(t) \quad \text { on } \quad \Gamma_{E} \times(0, T)
$$

where $C_{0}=\partial C / \partial t$ is also a real analytic function from $(0, T)$ with values in $\mathbb{R}$. In other words, for each $t$, $C_{0}(t)$ is a constant on $\Gamma_{E}$, in fact, it is the same constant on all the connected components of $\Gamma_{E}$. This will be important since in order to avoid this constant, we will take the tangential gradient $\nabla_{\tau}$ in (3.14) to obtain

$$
\nabla_{\tau} \frac{\partial}{\partial t} \sigma(\mathbf{z}, q) \mathbf{n} \cdot \mathbf{n}=0 \quad \text { on } \quad \Gamma_{E} \times(0, T)
$$

By analytic extension in time, we also have

$$
\nabla_{\tau} \frac{\partial}{\partial t} \sigma(\mathbf{z}, q) \mathbf{n} \cdot \mathbf{n}=0 \quad \text { on } \quad \Gamma_{E} \times(-\infty, T)
$$

On the other hand, we use the Fourier decomposition method for solving (3.12). Let

$$
V_{0}=\left\{\mathbf{z} \in H_{0}^{1}(\Omega)^{N}, \operatorname{div} \mathbf{z}=0\right\}
$$

and

$$
H_{0}=\left\{\mathbf{z} \in L^{2}(\Omega)^{N}, \operatorname{div} \mathbf{z}=0, \mathbf{z} \cdot \mathbf{n}=0 \text { on } \Gamma\right\},
$$

endowed with the usual inner products and norms of $H_{0}^{1}(\Omega)^{N}$ and $L^{2}(\Omega)^{N}$ respectively.

Let $\left(\mathbf{z}_{j}\right)_{j \geq 1}$ be an orthonormal basis in $H_{0}$ and orthogonal basis in $V_{0}$ of eigenfunctions of the Stokes operator associated with an increasing sequence of eigenvalues $\left(\lambda_{j}\right)_{j \geq 1}$ (see [14]):

$$
\begin{aligned}
-\nu \Delta \mathbf{z}_{j}+\nabla q_{j} & =\lambda_{j} \mathbf{z}_{j} \quad \text { in } \quad \Omega \\
\operatorname{div} \mathbf{z}_{j} & =0 \quad \text { in } \Omega \\
\mathbf{z}_{j} & =\mathbf{0} \quad \text { on } \Gamma \\
\left(\mathbf{z}_{j}, \mathbf{z}_{k}\right)_{H_{0}} & =\delta_{j k}, \quad\left(\mathbf{z}_{j}, \mathbf{z}_{k}\right)_{V_{0}}=0 \text { for } j \neq k .
\end{aligned}
$$


In order that $q_{j}$ is completely defined, we take

$$
\int_{\Omega} q_{j} d x=0
$$

We now take the decomposition of $\mathbf{z}_{T}$ on this basis

$$
\mathbf{z}_{T}=\sum_{j \geq 1} a_{j} \mathbf{z}_{j}
$$

Then

$$
\begin{aligned}
\mathbf{z}(t) & =\sum_{j \geq 1} a_{j} e^{-\lambda_{j}(T-t)} \mathbf{z}_{j} \\
q(t) & =\sum_{j \geq 1} a_{j} e^{-\lambda_{j}(T-t)} q_{j} \\
\sigma(\mathbf{z}, q) \mathbf{n} \cdot \mathbf{n} & =\sum_{j \geq 1} a_{j} e^{-\lambda_{j}(T-t)} \sigma\left(\mathbf{z}_{j}, q_{j}\right) \mathbf{n} \cdot \mathbf{n}
\end{aligned}
$$

and

$$
\nabla_{\tau} \sigma(\mathbf{z}, q) \mathbf{n} \cdot \mathbf{n}=\sum_{j \geq 1} a_{j} e^{-\lambda_{j}(T-t)} \nabla_{\tau} \sigma\left(\mathbf{z}_{j}, q_{j}\right) \mathbf{n} \cdot \mathbf{n}
$$

All these series are uniformly converging in time for $t \leq T-\delta, \delta>0$.

Let us rewrite the eigenvalues by considering only distinct eigenvalues (with, possibly, multiplicity greater that 1 ). We write $\left(\mu_{k}\right)_{k \geq 1}$ the strictly increasing sequence of eigenvalues of (3.12) and we define

$$
\begin{aligned}
\mathbf{z}_{\mu_{k}} & =\sum_{j, \lambda_{j}=\mu_{k}} a_{j} \mathbf{z}_{j} \\
q_{\mu_{k}} & =\sum_{j, \lambda_{j}=\mu_{k}} a_{j} q_{j},
\end{aligned}
$$

the projections of $\mathbf{z}$ and $q$ over the eigenspace associated to $\mu_{k}$. Then $\mathbf{z}_{\mu_{k}}=0$ if and only if $a_{j}=0, \forall j, \lambda_{j}=\mu_{k}$, and if $\mathbf{z}_{\mu_{k}} \neq 0$, it is an eigenfunction of the Stokes operator associated to $\mu_{k}$. We now have

$$
\nabla_{\tau}[\sigma(\mathbf{z}, q) \mathbf{n} \cdot \mathbf{n}]=\sum_{k \geq 1} e^{-\mu_{k}(T-t)} \nabla_{\tau}\left[\sigma\left(\mathbf{z}_{\mu_{k}}, q_{\mu_{k}}\right) \mathbf{n} \cdot \mathbf{n}\right] .
$$

As the eigenvalues $\mu_{k}$ are all distinct and positive, and due to the uniform convergence in time of the series, condition (3.16) implies

$$
\nabla_{\tau} \sigma\left(\mathbf{z}_{\mu_{k}}, q_{\mu_{k}}\right) \mathbf{n} \cdot \mathbf{n}=0 \quad \text { on } \quad \Gamma_{E} \quad \forall k \geq 1 .
$$

Therefore for every $k \geq 1, \sigma\left(\mathbf{z}_{\mu_{k}}, q_{\mu_{k}}\right) \mathbf{n} \cdot \mathbf{n}$ has to be constant on each connected component of $\Gamma_{E}$. In fact, because of the analyticity of $\sigma(\mathbf{z}, q) \mathbf{n} \cdot \mathbf{n}$ and from (3.14), for each $K$ these constants have to be the same on all of the connected components of $\Gamma_{E}$, that is to say

$$
\forall k \geq 0, \exists c_{k} \in \mathbb{R}, \quad \text { such that } \quad \sigma\left(\mathbf{z}_{\mu_{k}}, q_{\mu_{k}}\right) \mathbf{n} \cdot \mathbf{n}=c_{k} \quad \text { on } \quad \Gamma_{E} .
$$


Indeed, if $c_{k}$ and $c_{k}^{\prime}$ are two different constants on two different connected components of $\Gamma_{E}$, by substracting the corresponding associated series expansions of (3.14) on $\Gamma_{E}$, and by using that $C_{0}$ is the same in all the conected components of $\Gamma_{E}$, we obtain

$$
0=\sum_{k \geq 1} \mu_{k}\left(c_{k}-c_{k}^{\prime}\right) e^{-\mu_{k}(T-t)}, \forall t \in(-\infty, T),
$$

and this implies $c_{k}=c_{k}^{\prime}$.

Now, as $\operatorname{div} \mathbf{z}_{\mu_{k}}=0$ and $\mathbf{z}_{\mu_{k}}=0$ on $\Gamma$, we can notice that (see Appendix)

$$
\sigma\left(\mathbf{z}_{\mu_{k}}, q_{\mu_{k}}\right) \mathbf{n} \cdot \mathbf{n}=-q_{\mu_{k}} \quad \text { on } \quad \Gamma .
$$

Summarizing the information, we have, for every $k \geq 1$,

$$
\begin{aligned}
-\nu \Delta \mathbf{z}_{\mu_{k}}+\nabla q_{\mu_{k}} & =\mu_{k} \mathbf{z}_{\mu_{k}} \quad \text { in } \quad \Omega \\
\operatorname{div} \mathbf{z}_{\mu_{k}} & =0 \quad \text { in } \quad \Omega \\
\mathbf{z}_{\mu_{k}} & =0 \quad \text { on } \quad \Gamma
\end{aligned}
$$

and

$$
\sigma\left(\mathbf{z}_{\mu_{k}}, q_{\mu_{k}}\right) \mathbf{n} \cdot \mathbf{n}=-q_{\mu_{k}}=c_{k} \in \mathbb{R} \quad \text { on } \quad \Gamma_{E} .
$$

In order to show that $\mathbf{z}_{T}=\mathbf{0}$ we have to show that (3.28) and (3.29) imply $\mathbf{z}_{\mu_{k}}=0, \forall k \geq 1$, so that $a_{j}=0$, $\forall j \geq 1$.

This corresponds to a unique continuation property for the eigenvalue problem which will be treated in the next section.

Notice that up to now, among the specific hypothesis of Theorem 3.1, we have only used the hypothesis $\gamma=\Gamma_{E}$. The argument used to reduce the approximate controllability problem to a unique continuation one is valid for general dimension $N$ and for general geometries of $\Omega$.

Let us assume for the moment that the unique continuation property for the eigenvalue problem is valid, i.e. any solution $\mathbf{z}_{\mu_{k}}$ of (3.28) satisfying (3.29) must be 0 .

Then $\mathbf{z}_{T}=\mathbf{0}, \mathbf{z}=\mathbf{0}$ in $\Omega \times(0, T)$ and $q(t)=c(t)$ in $\Omega \times(0, T)$, so that

$$
\sigma(\mathbf{z}, q) \mathbf{n} \cdot \mathbf{n}=-c(t) .
$$

From (3.10) we have $\psi_{T}=0$ and $\varphi(t)=\varphi_{T}$, for all $t \leq T$. Now (3.7) gives

$$
\begin{aligned}
\mathbf{B} \varphi_{T} & =-c(t)=-c \in \mathbb{R} \quad \text { on } \quad \Gamma_{E} \\
\varphi_{T} & \in W_{0} .
\end{aligned}
$$

After multiplying by $\varphi_{T}$ and integrating on $\Gamma_{E}$ the equation above, from definition (3.1) of $W_{0}$ we easily deduce that $b\left(\varphi_{T}, \varphi_{T}\right)=0$, thus the coercivity hypothesis $(2.4)$ implies $\varphi_{T}=0$.

Therefore the unique continuation property for the eigenvalue problem implies approximate controllability for our original system $(2.5,2.6)$.

Remark 3.2. In the case $\gamma=\Gamma_{E}$ that we have considered, the system $(3.5,3.7,(3.10)$ is decoupled and therefore the problem under consideration is equivalent to the study of the approximate controllability for the Stokes system with boundary control acting on the normal component of the velocity on a part of the boundary. Let us mention that the complementary problem of the approximate controllability of the Stokes system with boundary control acting only on the tangential component of the velocity has been studied in [10]. 


\section{UNique CONTINUATION PROPERTY FOR THE EIGENVALUE PROBLEM}

The second part of the proof of Theorem 3.1 is given in this section by proving Theorem 4.1, a unique continuation result for (3.28-3.29) under the specific hypothesis of Theorem 3.1.

Let us consider the eigenvalue problem: find $(\mathbf{z}, q) \in V_{0} \times L^{2}(\Omega)$ and

$$
\begin{aligned}
-\nu \Delta \mathbf{z}+\nabla q & =\mu \mathbf{z} \quad \text { in } \quad \Omega \\
\operatorname{div} \mathbf{z} & =0 \quad \text { in } \quad \Omega \\
\mathbf{z} & =\mathbf{0} \quad \text { on } \quad \Gamma
\end{aligned}
$$

with the additional condition

$$
\sigma(\mathbf{z}, q) \mathbf{n} \cdot \mathbf{n}=-q=c \in \mathbb{R} \quad \text { on } \quad \Gamma_{E} .
$$

We want to prove that $(4.1,4.2)$ imply

$$
\mathbf{z}=\mathbf{0} \text { in } \Omega
$$

This is a unique continuation property for the eigenvalue problem.

Theorem 4.1. We suppose that $N=2, \Gamma_{R}$ and $\Gamma_{E}$ are separately regular and one junction point between $\Gamma_{R}$ and $\Gamma_{E}$, that we call $A$, is an exact rectangular corner, i.e. there exists a neighborhood $\mathbf{U}$ of $A$ such that $\left(\Gamma_{E} \cap \mathbf{U}\right)$ and $\left(\Gamma_{R} \cap \mathbf{U}\right)$ are the two sides of a rectangular angle with vertex $A$.

Then any solution $\mathbf{z}$ of $(4.1,4.2)$ verifies $\mathbf{z}=\mathbf{0}$ in $\Omega$.

Proof. As $\operatorname{div} \mathbf{z}=0$ and $\Omega$ is a $2 d$ connected domain, we can introduce the stream function (cf. [14]). Let $\psi$ be such that $\mathbf{z}=\left(\frac{\partial \psi}{\partial x_{2}},-\frac{\partial \psi}{\partial x_{1}}\right)$. Condition $\mathbf{z}=0$ on $\Gamma$ implies that $\nabla \psi=0$ on $\Gamma$, that is to say $\frac{\partial \psi}{\partial \mathbf{n}}=0$ and $\psi$ constant on each connected component of $\Gamma$. We take the choice $\psi=0$ on $\Gamma$. Moreover $-\Delta \psi=\frac{\partial z_{2}}{\partial x_{1}}-\frac{\partial z_{1}}{\partial x_{2}}$ in $\Omega$. Therefore, it is easy to see that $\psi$ is also the unique solution of the following biharmonic problem:

$$
\begin{aligned}
\Delta^{2} \psi & =-\mu \Delta \psi \quad \text { in } \quad \Omega \\
\psi & =0 \quad \text { on } \quad \Gamma \\
\frac{\partial \psi}{\partial \mathbf{n}} & =0 \quad \text { on } \quad \Gamma
\end{aligned}
$$

with an additional boundary condition. This condition can be formally obtained by taking the tangential component of the trace on the boundary of equation (4.1a) and by using the supplementary condition (4.2). In this way we obtain:

$$
\frac{\partial}{\partial \mathbf{n}} \Delta \psi=0 \quad \text { on } \quad \Gamma_{E}
$$

Without loss of generality, we can assume, taking $O x$ and $O y$ as rectangular axis, that $A$ is the origin $O$, $\mathbf{U}$ is a ball centered at the origin, $\left(\Gamma_{E} \cup \mathbf{U}\right)$ is supported by $O x,\left(\Gamma_{R} \cap \mathbf{U}\right)$ is supported by $O y$ and $\Omega \cap \mathbf{U}$ is contained in

$$
\left\{(x, y) \in \mathbb{R}^{2}, x>0, y>0\right\}
$$

Let us write

$$
\begin{aligned}
\widetilde{\mathbf{U}} & =\mathbf{U} \cap\left\{(x, y) \in \mathbb{R}^{2}, x>0\right\} \\
\widetilde{\Gamma}_{R} & =\left\{(x, y) \in \mathbb{R}^{2}, x=0\right\} \cap \mathbf{U}
\end{aligned}
$$




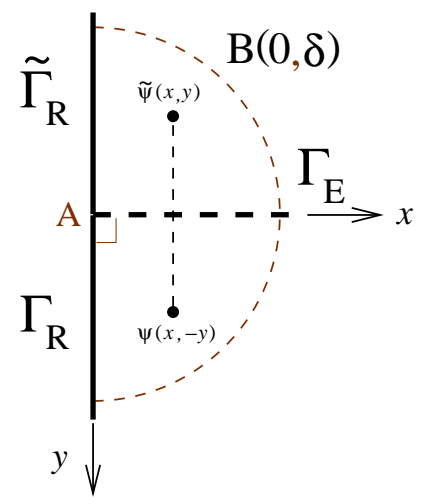

FiguRE 2. Fig. 2. Extension by parity in the proof of Theorem 4.1.

and let us extend $\psi$ by parity to $\widetilde{\mathbf{U}}$ by

$$
\widetilde{\psi}(x, y)=\left\{\begin{array}{ccc}
\psi(x, y) & \text { if } & (x, y) \in \widetilde{\mathbf{U}}, y \geq 0 \\
\psi(x,-y) & \text { if } & (x, y) \in \widetilde{\mathbf{U}}, y<0 .
\end{array}\right.
$$

Then $\Delta \widetilde{\psi}$ is "continuous" across $\Gamma_{E} \cap \mathbf{U}$ and because of (4.4) we have

$$
\begin{aligned}
\Delta^{2} \widetilde{\psi} & =-\mu \Delta \widetilde{\psi} & \text { in } \quad \widetilde{\mathbf{U}} \\
\widetilde{\psi} & =0 \quad \text { on } & \widetilde{\Gamma}_{R} \\
\frac{\partial}{\partial \mathbf{n}} \widetilde{\psi} & =0 \quad \text { on } & \widetilde{\Gamma}_{R} .
\end{aligned}
$$

Therefore there exists a ball $B(0, \delta)$ such that $\widetilde{\psi}$ is real analytic in $B(0, \delta) \cap\left\{(x, y) \in \mathbb{R}^{2}, x \geq 0\right\}$ (see [8], Vol. III, p. 32, Th. 1.3).

Now we can expand $\psi$ in power series of $x$ and $y$ in $\Omega \cap B(0, \delta)$ :

$$
\psi(x, y)=\sum_{i, j \geq 0} a_{i j} x^{i} y^{j} .
$$

From (4.3) we obtain for each $i, j \geq 0$ :

$$
\begin{gathered}
(i+4)(i+3)(i+2)(i+1) a_{i+4, j}+2(i+2)(i+1)(j+2)(j+1) a_{i+2, j+2} \\
+(j+4)(j+3)(j+2)(j+1) a_{i, j+4}-\mu\left[(i+2)(i+1) a_{i+2, j}+(j+2)(j+1) a_{i, j+2}\right] . \\
a_{i, 0}=0 ; a_{i, 1}=0 \quad \forall i \geq 0 \\
a_{0, j}=0 ; a_{1, j}=0 \quad \forall j \geq 0 .
\end{gathered}
$$

And (4.4) gives

$$
a_{i, 3}=0 \quad \forall i \geq 0 .
$$

From $(4.7,4.8)$ and $(4.10)$ we easily deduce

$$
a_{i, 2 k+1}=0 \quad \forall i \geq 1, \forall k \geq 0 .
$$


Now, using $(4.8,4.9)$ and taking $i=0, j=0$, then $i=1, j=0$ in (4.7) we obtain

$$
\begin{aligned}
& a_{2,2}=0 \\
& a_{3,2}=0 .
\end{aligned}
$$

Let us assume the following recurrence hypothesis

$$
\begin{aligned}
a_{2 k, 2} & =a_{2(k-1), 4}=\ldots=a_{2,2 k}=0 \\
a_{2 k+1,2} & =a_{2 k-1,4}=\ldots=a_{3,2 k}=0 .
\end{aligned}
$$

Notice that we have just shown in $(4.12,4.13)$ that $(4.14,4.15)$ are valid for $k=1$. Let us show that we have

$$
\begin{aligned}
& a_{2 k+2,2}=a_{2 k, 4}=\ldots=a_{2,2(k+1)}=0 \\
& a_{2 k+3,2}=a_{2 k+1,4}=\ldots=a_{3,2(k+1)}=0 .
\end{aligned}
$$

By multiplying both sides by $i ! j$ ! we rewrite $(4.7)$ as

$$
\begin{aligned}
(i+4) ! j ! a_{i+4, j} & +2(i+2) !(j+2) ! a_{i+2, j+2}+i !(j+4) ! a_{i, j+4} \\
& =-\mu\left[(i+2) ! j ! a_{i+2, j}+i !(j+2) ! a_{i, j+2}\right] \quad \forall i, j \geq 0 .
\end{aligned}
$$

We take successively in (4.18):

$$
i=2 k, j=0 ; i=2(k-1), j=2 ; \ldots ; i=0, j=2 k .
$$

Using $(4.8,4.9,4.14)$ we obtain the following $(k+1) \times(k+1)$ linear system (notice that all right hand sides of (4.18) are zero):

$$
M_{k+1} A_{k+1}=0,
$$

where

$$
A_{k+1}=\left(\begin{array}{c}
a_{2 k+2,2} \\
\vdots \\
a_{2,2 k+2}
\end{array}\right)
$$

and

$$
M_{k+1}=\left[\begin{array}{ccccc}
2(2 k+2) ! 2 ! & (2 k) ! 4 ! & & & \\
(2 k+2) ! 2 ! & 2(2 k) ! 4 ! & (2 k-2) ! 6 ! & & \\
& (2 k) ! 4 ! & 2(2 k-2) ! 6 ! & (2 k-4) ! 8 ! & \\
& \ddots & \ddots & \ddots & \\
& & \ddots & \ddots & \ddots \\
& & 6 !(2 k-2) ! & 2 \cdot 4 !(2 k) ! & 2 !(2 k+2) ! \\
& & & 4 !(2 k) ! & 2 \cdot 2 !(2 k+2) !
\end{array}\right] .
$$

Then

$$
\operatorname{det} M_{k+1}=[(2 k+2) ! 2 !][(2 k) ! 4 !] \ldots[2 !(2 k+2) !] \Delta_{k+1},
$$


where $\Delta_{k+1}$ is the following $(k+1) \times(k+1)$ determinant:

$$
\Delta_{k+1}=\left|\begin{array}{cccccc}
2 & 1 & & & & \\
1 & 2 & 1 & & & \\
& \ddots & \ddots & \ddots & & \\
& & \ddots & \ddots & \ddots & \\
& & & 1 & 2 & 1 \\
& & & & 1 & 2
\end{array}\right|
$$

It is easy to see by induction that $\Delta_{k+1}=k+2$, since $\Delta_{k+1}=2 \Delta_{k}-\Delta_{k-1}$ and $\Delta_{1}=2$. Therefore

$$
A_{k+1}=\left(\begin{array}{c}
0 \\
\vdots \\
0 \\
\vdots \\
0
\end{array}\right)
$$

which proves (4.16).

Now we take successively in (4.18):

$$
i=2 k+1, j=0 ; i=2 k-1, j=2 ; \ldots ; i=1, j=2 k
$$

and we obtain, using (4.8), (4.9) and (4.15):

$$
N_{k+1} B_{k+1}=0,
$$

where

$$
B_{k+1}=\left(\begin{array}{c}
a_{2 k+3,2} \\
\vdots \\
a_{3,2 k+2}
\end{array}\right)
$$

and

$$
N_{k+1}=\left[\begin{array}{ccccc}
2(2 k+3) ! 2 ! & (2 k+1) ! 4 ! & & & \\
(2 k+3) ! 2 ! & 2(2 k+1) ! 4 ! & (2 k-1) ! 6 ! & & \\
& \ddots & \ddots & \ddots & \\
& & \ddots & \ddots & \ddots \\
& & 7 !(2 k-2) ! & 2 \cdot 5 !(2 k) ! & 3 !(2 k+2) ! \\
& & & 5 !(2 k) ! & 2 \cdot 3 !(2 k+2) !
\end{array}\right] .
$$

We have here

$$
\operatorname{det} N_{k+1}=[(2 k+3) ! 2 !][(2 k+1) ! 4 !] \ldots[3 !(2 k+2) !] \Delta_{k+1},
$$

so that we obtain

$$
B_{k+1}=\left(\begin{array}{c}
0 \\
\vdots \\
0 \\
\vdots \\
0
\end{array}\right)
$$

which proves (4.17). 
Therefore, we have

$$
a_{i, 2 k}=0 \quad \forall i \geq 0, \forall k \geq 0
$$

which, together with (4.11), proves that

$$
\psi=0 \text { in } \Omega
$$

and

$$
\mathbf{z}=0, q=\text { cte in } \Omega,
$$

and the proof of Theorem 4.1 is complete.

\section{Comments And COnJeCtures}

The previous proof uses all conditions (4.3) and (4.4) locally but in the neighborhood of a rectangular corner $\Gamma$. In addition, it uses the real analyticity of $\psi$ in that neighborhood.

As already mentioned, the counterexample in the case of a ball given in [9] shows that it is hopeless to use a local argument in the neighborhood of a regular point of $\Gamma$.

The hypothesis saying that the junction between $\Gamma_{E}$ and $\Gamma_{R}$ is an exact rectangular corner seems purely technical but at the moment we do not know how to extend the proof of Theorem 4.1 to other cases.

Extensions of results of [6] on singularities can perhaps be used to prove analyticity in the neighborhood of convex corners. The $3 d$-case has to be treated directly on the Stokes system and the geometric conditions are much more difficult to write.

Nevertheless, our conjecture is that Theorem 4.1 is valid if $\Gamma$ is not analytic (a first step would be to prove it when $\Gamma$ has a convex corner).

A second conjecture would be that, when $\Gamma$ is analytic, the only counterexample to uniqueness property above is the case of a ball. The counterexample in which $\Omega$ is a ball corresponds to the existence of an eigenfunction for the Stokes operator which induces a constant pressure on a part of the boundary. There is of course a strong relation between our problem and the ones treated by Berenstein [1,2], Beretta and Vogelius [3], Vogelius [15], Williams [16] and others $[5,13]$ related to the Pompeiu problem.

\section{ApPEndix}

Proposition 6.1. Let $\Omega$ be a bounded connected open set in $\mathbb{R}^{N}(N=2$ or 3 ) with Lipschitz boundary $\Gamma$ and unit exterior normal $\mathbf{n}$. Let $\mathbf{z} \in H^{2}(\Omega)^{N} \cap H_{0}^{1}(\Omega)^{N}$ with $\operatorname{div} \mathbf{z}=0$. Then

$$
(\nabla \mathbf{z}) \mathbf{n} \cdot \mathbf{n}=0 \quad \text { on } \quad \Gamma .
$$

Proof. We take the standard notation of implicit summation of repeated indices. We do the proof for $N=2$, the proof for the case $N=3$ is very similar. We will use the normal $\mathbf{n}=\left(n_{1}, n_{2}\right)$ and the tangent $\tau=\left(\tau_{1}, \tau_{2}\right)=$ $\left(-n_{2}, n_{1}\right)$ on $\Gamma$. We can prove the result for $\mathbf{z} \in H^{3}(\Omega)^{2} \cap H_{0}^{1}(\Omega)^{2}$ and then use a density argument. We take a test function $\phi \in H^{1}(\Omega)^{2}$. From $\operatorname{div} \mathbf{z}=0$ it is clear that

$$
\begin{aligned}
0 & =\int_{\Omega} \frac{\partial}{\partial x_{j}}\left(\frac{\partial z_{i}}{\partial x_{i}}\right) \cdot \phi_{j} d x \\
& =\int_{\Omega} \frac{\partial}{\partial x_{i}}\left(\frac{\partial z_{i}}{\partial x_{j}}\right) \cdot \phi_{j} d x \\
& =\int_{\Gamma} \frac{\partial z_{i}}{\partial x_{j}} n_{i} \phi_{j} d \Gamma-\int_{\Omega} \frac{\partial z_{i}}{\partial x_{j}} \frac{\partial \phi_{j}}{\partial x_{i}} d x \\
& =\int_{\Gamma}\left(\frac{\partial z_{i}}{\partial x_{j}} n_{i} n_{j}\right)\left(\phi_{j} n_{j}\right) d \Gamma+\int_{\Gamma}\left(\frac{\partial z_{i}}{\partial x_{j}} n_{i} \tau_{j}\right)\left(\phi_{j} \tau_{j}\right) d \Gamma-\int_{\Omega} \frac{\partial z_{i}}{\partial x_{j}} \frac{\partial \phi_{j}}{\partial x_{i}} d x
\end{aligned}
$$


Since $\mathbf{z}=0$ on $\Gamma$ we have $\frac{\partial z_{i}}{\partial x_{j}} \tau_{j}=0$ and then

$$
\int_{\Gamma}\left(\frac{\partial z_{i}}{\partial x_{j}} n_{i} n_{j}\right)\left(\phi_{j} n_{j}\right) d \Gamma-\int_{\Omega} \frac{\partial z_{i}}{\partial x_{j}} \frac{\partial \phi_{j}}{\partial x_{i}} d x=0, \quad \forall \phi \in H^{1}(\Omega)^{2} .
$$

Taking first a test function $\phi$ with $\operatorname{div} \phi=0$ in $\Omega$ we obtain from (6.2) that

$$
\int_{\Gamma}\left(\frac{\partial z_{i}}{\partial x_{j}} n_{i} n_{j}\right) g d \Gamma=0, \quad \forall g \in L^{2}(\Gamma), \int_{\Gamma} g d \Gamma=0,
$$

that is to say

$$
\frac{\partial z_{i}}{\partial x_{j}} n_{i} n_{j}=c \quad \text { on } \quad \Gamma
$$

where $c$ is a constant on $\Gamma$. Taking then $\phi(\mathbf{x})=\mathbf{x}$ we obtain from (6.2) that

$$
0=\int_{\Gamma}\left(\frac{\partial z_{i}}{\partial x_{j}} n_{i} n_{j}\right)\left(x_{j} n_{j}\right) d \Gamma=c \int_{\Gamma} x_{j} n_{j} d \Gamma=c N \operatorname{meas}(\Omega),
$$

and this implies $c=0$.

\section{REFERENCES}

[1] C. Berenstein, An inverse spectral theorem and its relation to the Pompeiu problem. J. Anal. Math. 37 (1980) $128-144$.

[2] C. Berenstein, The Pompeiu problem, what's new?, Deville R. et al. (Ed.), Complex analysis, harmonic analysis and applications. Proceedings of a conference in honour of the retirement of Roger Gay, June 7-9, 1995, Bordeaux, France. Harlow: Longman. Pitman Res. Notes Math. Ser. 347 (1996) 1-11.

[3] E. Beretta and M. Vogelius, An inverse problem originating from magnetohydrodynamics. III: Domains with corners of arbitrary angles. Asymptotic Anal. 11 (1995) 289-315.

[4] H. Brezis, Analyse Fonctionnelle, Théorie et Applications, Collection Math. Appl. Pour la Maîtrise, Masson, Paris (1983).

[5] L. Brown, B.M. Schreiber and B.A. Taylor, Spectral synthesis and the Pompeiu problem. Ann. Inst. Fourier 23 (1973) $125-154$.

[6] P. Grisvard, Elliptic problems in nonsmooth domains. Monographs and Studies in Mathematics, 24. Pitman, Boston-LondonMelbourne (1985).

[7] J.-L. Lions, Remarques sur la contrôlabilité approchée, Control of distributed systems, Span.-Fr. Days, Malaga/Spain 1990, Grupo Anal. Mat. Apl. Univ. Malaga 3 (1990) 77-87.

[8] J.-L. Lions and E. Magenes, Problèmes Aux Limites Non Homogènes et Applications, Vols. I, II, III, Dunod, Paris (1968).

[9] J.-L. Lions and E. Zuazua, Approximate controllability of a hydro-elastic coupled system. ESAIM: Contr. Optim. Calc. Var. 1 (1995) 1-15.

[10] A. Osses, A rotated direction multiplier technique. Applications to the controllability of waves, elasticity and tangential Stokes control, SIAM J. Cont. Optim., to appear.

[11] A. Osses and J.-P. Puel, Approximate controllability of a linear model in solid-fluid interaction in a rectangle. to appear.

[12] A. Pazy, Semigroups of linear operators and applications to partial differential equations, Springer-Verlag, New York. Appl. Math. Sci. 44 (1983).

[13] J. Serrin, A symmetry problem in potential theory. Arch. Rational. Mech. Anal. 43 (1971) 304-318.

[14] R. Temam, Navier-Stokes Equations, North-Holland, Amsterdam (1977).

[15] M. Vogelius, An inverse problem for the equation $\Delta u=-c u-d$. Ann. Inst. Fourier, 44 (1994) 1181-1209.

[16] S.A. Williams, Analyticity of the boundary for Lipschitz domains without the Pompeiu property. Indiana Univ. Math. J. 30 (1981) 357-369. 